\title{
Maternal and Fetal Outcome of Fever in Pregnancy
}

\author{
Shwetha Surya Raj ${ }^{1}$, M. Sarmishta ${ }^{2}$, M.G. Dhanalakshmi ${ }^{3}$ \\ 1, 2, 3 Department of Obstetrics and Gynaecology, Sri Ramachandra Medical University, \\ Chennai, Tamil Nadu, India.
}

\section{ABSTRACT}

\section{BACKGROUND}

This study was conducted to evaluate the maternal and fetal outcome of fever in pregnancy.

\section{METHODS}

The present study was a prospective observational study conducted from April 2012 - March 2014 among patients in the Department of Obstetrics and Gynaecology, Sri Ramachandra Institute of Higher Education and Research Institute, and they were followed up to the time of delivery to determine the maternal and foetal outcome. This is a prospective study and sample size was 120 patients. All patients admitted with fever irrespective of the gestational age and parity were included in the study. The ethical committee of Ramachandra Medical College and Hospital approved the study. Reference CSP-MED / 13 / AUG / 08 / 47.

\section{RESULTS}

Fever in pregnancy nearing term has been associated with adverse outcomes. Even elevated temperature in the first trimester can lead to fetal loss. Urinary tract infection was the most common infection during pregnancy. E. coli was the most common organism isolated. Other aetiologies encountered were dengue, malaria, scrub typhus, leptospirosis, enteric fever, H1N1, Influenza A viral fever, respiratory tract infections, and chicken pox. The most frequently seen adverse outcome in this study was abortion. There was one maternal mortality due to H1N1 and one neonatal mortality due to dengue haemorrhagic fever which was transmitted to the fetus. There was one fetus with multiple anomalies and the mother had a history of chicken pox in the first trimester. There were 15 cases of premature rupture of membrane and 12 cases had preterm labour. Intrapartum fever was associated with postpartum haemorrhage and meconium-stained liquor in some mothers.

\section{CONCLUSIONS}

Fever during pregnancy can potentially lead to adverse maternal and perinatal outcomes even at a low-grade temperature of a short duration of less than a week. Even elevated temperature in the first trimester can lead to fetal loss. Fever in pregnancy nearing term has been associated with adverse perinatal outcomes. Some virulent organisms can lead to fetal anomalies and maternal death.

\section{KEY WORDS}

Fever in Pregnancy, Maternal Outcome, Foetal Outcome
Corresponding Author: Dr. M. Sarmishta,

No 1, Somasundaram Street, T.Nagar, Chennai - 600017, Tamil Nadu, India. E-mail: sarmishta83@yahoo.co.in

DOI: $10.14260 / \mathrm{jemds} / 2021 / 200$

How to Cite This Article:

Raj SS, Sarmishta M, Dhanalakshmi MG. Maternal and fetal outcome of fever in pregnancy. J Evolution Med Dent Sci 2021;10(13):925-929, $10.14260 /$ jemds $/ 2021 / 200$

Submission 12-11-2020,

Peer Review 30-01-2021,

Acceptance 06-02-2021,

Published 29-03-2021.

Copyright (C) 2021 Shwetha Surya Raj et al. This is an open access article distributed under Creative Commons Attribution License [Attribution 4.0 International (CC BY 4.0)] 


\section{BACKGROUND}

Fever is an elevation in body temperature above the normal range which occurs due to the change in the temperature set point of the thermoregulatory centre in the anterior hypothalamus called the preoptic area. Fever in pregnancy poses as a threat to both the mother and the fetus and is of much concern to the treating obstetrician. Fever is a response of the host's immune system to any infection and manifests as an elevation in body temperature. A number of aetiologies of fever in pregnancy have been studied like urinary tract infections, respiratory tract infections, typhoid, malaria, dengue, leptospirosis, scrub typhus, influenza virus, chicken pox etc. and have been associated with adverse outcomes depending on the severity of the infection, period of infection and duration of the illness. As pregnant women are generally susceptible to infections, early diagnosis and treatment can prevent adverse outcomes.

The placenta does act as a mechanical barrier to certain infections; however, most viral infections can cross the placenta and affect the fetus. Hyperthermia acts as a teratogen, an agent or factor which causes malformation of an embryo, in some animals where it can induce resorption of the fetus and fetal death. Fever during pregnancy, especially in the period of embryogenesis, is also suspected as being a risk factor for fetal death in human beings. ${ }^{1}$ Risks of spontaneous abortion, intrauterine growth restriction, anomalous fetus, intrauterine death, preterm labour, premature rupture of membranes, neonatal sepsis, meconium stained liquor, postpartum haemorrhage, maternal sepsis and even maternal and fetal death have been associated with fever in pregnancy. ${ }^{2}$ Fever or pyrexia is defined as an elevation in body temperature above $98.6^{\prime} \mathrm{F}$. It is one of the array of host defense responses to invasion of the body by microbes. It can be associated with constitutional symptoms like headache, fatigue and generalised body pains. ${ }^{2}$ Fever in pregnancy has been associated with adverse outcomes. ${ }^{3}$ Hence early diagnosis and management can prevent the same. An acute rise in body temperature usually occurs when there is an in increase in hormone levels of adrenocorticotrophic hormone, growth hormone and in increase in neutrophils, muscle proteolysis and lipogenesis.

Thermoregulation occurs at the preoptic area of the hypothalamus. It is circadian in nature; lowest in the early morning hours and highest at 4-6 PM. Body temperature fluctuates with illness as well. Fever arises from a complex sequence of interactions among soluble factors and host cells, initiated in periphery and transmitted to brain (preoptic area of hypothalamus) to modulate a fever response. A number of endogenous inflammatory factors like interleukin- $1 \beta$, tumour necrosis factor \& iinterleukin- 6 etc., are released in response to exogenous pyrogens (microbes) and directly provoke a febrile response. 4 These cytokines bind to their own receptors in close relation to the preoptic area of the anterior hypothalamus. This activates the enzyme phospholipase A2 causing the release of arachidonic acid from the plasma membrane which acts as a substrate for the cyclooxygenase pathway. Arachidonic acid diffuses through the blood brain barrier and decreases the rate of firing of the preoptic warm sensitive neurons causing a decrease in heat loss and increase in heat generation. Some cytokines increase the cyclooxygenase expression directly and cause a release in prostaglandin E2 which causes a rise in body temperature. Increase in sympathetic activity can also cause hyperthermia. Urinary tract infection is one of the most common causes of fever in pregnancy occurring at any trimester. ${ }^{4}$ This is due to the alteration in the anatomical structure of the urinary tract. Dilatation of the renal pelvis and ureters during pregnancy is due to the dextrorotation of the uterus causing compression of the ureters.

Vaginal infection with Group B streptococcus is known to be a cause of neonatal sepsis and is associated with preterm rupture of membranes, and preterm labour and delivery. Group b streptococcus (GBS) is found to be the causative organism of urinary tract infections in approximately 5 percent of patients. The organisms causing urinary tract infections during pregnancy are the same as those found in non-pregnant patients. 80 to 90 percent of infections are due to Escherichia coli. ${ }^{5}$ Other gram-negative rods such as Proteus mirabilis and Klebsiella pneumonia are also commonly seen. Gram-positive organisms such as group B streptococcus and Staphylococcus saprophyticus are less common causes of urinary tract infections. Less common organisms that may cause urinary tract infections (UTI) include enterococci, Gardnerella vaginalis and urea plasma urealyticum. Significant bacteriuria may exist in asymptomatic patients. In the 1960's, significant bacteriuria has been historically defined as finding more than $10^{5}$ colony-forming units per $\mathrm{mL}$ of urine. Urinary tract infections can be asymptomatic or symptomatic leading to the development of acute cystitis or pyelonephritis. Recent studies of women with acute dysuria have shown the presence of significant bacteriuria with lower colony counts. This has not been studied in pregnant women and finding more than $10^{5}$ colony-forming units per $\mathrm{mL}$ of urine remains the commonly accepted standard. Screening helps in drastically decreasing the incidence of cystitis and pyelonephritis in pregnancy. ${ }^{6}$ Also some studies said that by treating asymptomatic bacteriuria, the incidence of preterm birth and low birth weight infants decreased.

The American College of Obstetrics and Gynaecology recommends that a urine culture should be obtained at the first antenatal visit. A repeat urine culture should be obtained during the third trimester, as the urine of treated patients may not remain sterile for the entire pregnancy. By screening for and aggressively treating pregnant women with asymptomatic bacteriuria, it is possible to significantly decrease the annual incidence of pyelonephritis during pregnancy. Acute cystitis is differentiated from asymptomatic bacteriuria by the presence of symptoms such as dysuria, urgency and frequency in febrile patients with no evidence of systemic illness. Up to 30 percent of patients with untreated asymptomatic bacteriuria later develop symptomatic cystitis. Acute pyelonephritis during pregnancy is a dreaded systemic illness that can progress to maternal sepsis, preterm labour and premature delivery. ${ }^{7}$ The diagnosis is made when the presence of bacteriuria is present along with systemic symptoms or signs such as fever, chills, nausea, vomiting and flank pain with or without symptoms of lower urinary tract infection (i.e., frequency and dysuria). ${ }^{8}$ Pyelonephritis occurs in 2 percent of pregnant women; up to 23 percent of these women may have a recurrence during the same pregnancy. This study was conducted to know the maternal and fetal outcome of fever in pregnancy. 


\section{METHODS}

The present study was a prospective observational study conducted from April 2012 - March 2014 on the patients in the Department of Obstetrics and Gynecology, Sri Ramachandra Medical College and Research Institute and they were followed up to the time of delivery to determine the maternal and foetal outcome. Sample size was 120 patients.

The ethical committee of Ramachandra Medical College and Hospital approved the study. Reference CSP-MED / 13 / AUG / 08 / 47. Before recruiting the patients for the study, an informed consent was taken from the patients.

Patients were followed up to the time of delivery to determine the following. Out of the 120 women, the number of women having various infections according to the aetiology of fever, duration of fever, onset of fever and maternal and fetal adverse outcomes of fever were studied.

- Maternal outcome

- Fetal outcome

All patients admitted with fever irrespective of the gestational age and parity were included in the study.

\section{Exclusion Criteria}

- Rheumatic heart disease

- Infective endocarditis

- Chorioamnionitis

- Premature rupture of membranes (PROM) / preterm premature rupture of membranes (PPROM)

- Post transfusion fever

\section{Statistical Analysis}

The aetiology of fever, adverse outcomes with the onset of fever, duration of fever, temperature, from the onset of fever and the interval of adverse outcomes were analysed. The Pvalue was calculated with chi-square test. The statistical software used was Statistical Package for the Social Sciences (SPSS)-16 Statistical Product and Service Solution.

\section{RESULTS}

\begin{tabular}{|ccc|}
\hline Aetiology & Frequency & Percent \\
Unknown aetiology & 36 & $30 \%$ \\
UTI & 32 & $26.7 \%$ \\
Uri & 21 & $17.5 \%$ \\
Dengue & 12 & $10 \%$ \\
Typhoid & 6 & $5.0 \%$ \\
Scrub typhus & 5 & $4.2 \%$ \\
H1n1 & 2 & $1.7 \%$ \\
Malaria & 2 & $1.7 \%$ \\
Leptospirosis & 2 & $1.7 \%$ \\
Pneumonia & 1 & $0.8 \%$ \\
Chicken pox & 1 & $0.8 \%$ \\
Total & $\mathbf{1 2 0}$ & $\mathbf{1 0 0} \%$ \\
\hline \multicolumn{2}{c}{} \\
\hline
\end{tabular}

$26.7 \%$ of the patients had urinary tract infection, and the most common organism involved was Escherichia coli followed by enterococcus.

\begin{tabular}{|c|c|c|c|c|}
\hline Outcome & $\begin{array}{c}\text { Ist }^{\text {st }} \\
\text { trimester }\end{array}$ & $\begin{array}{c}\text { II }^{\text {nd }} \\
\text { trimester }\end{array}$ & $\begin{array}{c}\text { III }^{\text {rd }} \\
\text { trimester }\end{array}$ & Total (\%) \\
\hline Abortion & 9 & 7 & 0 & $16(13.3 \%)$ \\
\hline Anomaly & 1 & 0 & 0 & $1(0.8 \%)$ \\
\hline IUD & 0 & 1 & 1 & $2(1.7 \%)$ \\
\hline IUGR & 0 & 2 & 5 & $7(5.8 \%)$ \\
\hline PROM & 0 & 1 & 14 & 15 (12.5\%) \\
\hline Preterm labour & 1 & 5 & 6 & $12(10 \%)$ \\
\hline PPH & 0 & 0 & 3 & $3(2.5 \%)$ \\
\hline MSL & 0 & 0 & 6 & $6(5 \%)$ \\
\hline Neonatal death & 0 & 0 & 1 & $1(0.8 \%)$ \\
\hline Neonatal sepsis & 0 & 0 & 9 & $9(7.5 \%)$ \\
\hline Maternal sepsis & 1 & 0 & 2 & $3(2.5 \%)$ \\
\hline Maternal mortality & 0 & 0 & 1 & $1(0.8 \%)$ \\
\hline Total & 12 & 16 & 48 & $76(63.3 \%)$ \\
\hline \multicolumn{5}{|c|}{ Table 2. Adverse Outcome with Onset of Fever } \\
\hline
\end{tabular}

Most of the adverse outcomes had the onset of fever in the third trimester. P-value of 0.001 which is highly significant.

\begin{tabular}{|c|c|c|c|c|}
\hline Outcome & 1 to 5 Days & 6 to 10 Days & > 10 Days & Total (\%) \\
\hline Abortion & 8 & 7 & 1 & $16(13.3 \%)$ \\
\hline Anomaly & 0 & 1 & 0 & $1(0.8 \%)$ \\
\hline IUD & 1 & 1 & 0 & $2(1.7 \%)$ \\
\hline IUGR & 3 & 4 & 0 & 7 (5.8\%) \\
\hline PROM & 14 & 1 & 0 & $15(12.5 \%)$ \\
\hline Preterm labour & 6 & 5 & 1 & $12(10 \%)$ \\
\hline PPH & 3 & 0 & 0 & $3(2.5 \%)$ \\
\hline MSL & 6 & 0 & 0 & $6(5 \%)$ \\
\hline Neonatal death & 0 & 1 & 0 & $1(0.8 \%)$ \\
\hline Neonatal sepsis & 7 & 2 & 0 & $9(7.5 \%)$ \\
\hline Maternal sepsis & 1 & 2 & 0 & $3(2.5 \%)$ \\
\hline Maternal mortality & 0 & 1 & 0 & $1(0.8 \%)$ \\
\hline Total & 49 & 25 & 2 & $76(63.3 \%)$ \\
\hline
\end{tabular}

49 (40.8\%) of the adverse outcomes occurred with fever of duration less than 6 days. However, this is not statistically significant.

\begin{tabular}{|c|c|c|c|c|c|c|c|}
\hline Outcome & $\begin{array}{l}98.7- \\
99.6 ' \mathrm{~F}\end{array}$ & $\begin{array}{c}99.7- \\
100.6 ' F\end{array}$ & $\begin{array}{c}100.7- \\
101.6 ' F\end{array}$ & $\begin{array}{c}101.7 \\
- \\
102.6^{\prime} \\
\text { F }\end{array}$ & $\begin{array}{l}102.7- \\
103.6^{\prime} \mathrm{F}\end{array}$ & $\stackrel{>}{103.7^{\prime} \mathrm{F}}$ & $\begin{array}{c}\text { Total } \\
\text { (\%) }\end{array}$ \\
\hline Abortion & 4 & 2 & 3 & 3 & 3 & 1 & $\begin{array}{c}16(13.3 \\
\%)\end{array}$ \\
\hline IUGR & 1 & 3 & 1 & 1 & 0 & 1 & $7(5.8 \%)$ \\
\hline PROM & 4 & 6 & 3 & 1 & 0 & 1 & $\begin{array}{c}15(12.5 \\
\%)\end{array}$ \\
\hline $\begin{array}{c}\text { Preterm } \\
\text { labour }\end{array}$ & 2 & 4 & 4 & 2 & 0 & 0 & $12(10 \%)$ \\
\hline PPH & 1 & 2 & 0 & 0 & 0 & 0 & $3(2.5 \%)$ \\
\hline MSL & 2 & 2 & 2 & 0 & 0 & 0 & 6 (5\%) \\
\hline $\begin{array}{c}\text { Neonatal } \\
\text { death }\end{array}$ & 0 & 0 & 1 & 0 & 0 & 0 & $1(0.8 \%)$ \\
\hline $\begin{array}{c}\text { Neonatal } \\
\text { sepsis }\end{array}$ & 2 & 2 & 3 & 0 & 0 & 2 & $9(7.5 \%)$ \\
\hline IUD & 1 & 0 & 1 & 0 & 0 & 0 & $2(1.7 \%)$ \\
\hline Anomaly & 0 & 1 & 0 & 0 & 0 & 0 & $1(0.8 \%)$ \\
\hline $\begin{array}{c}\text { Maternal } \\
\text { sepsis }\end{array}$ & 0 & 0 & 2 & 0 & 0 & 1 & $3(2.5 \%)$ \\
\hline $\begin{array}{l}\text { Maternal } \\
\text { mortality }\end{array}$ & 0 & 0 & 1 & 0 & 0 & 0 & $1(0.8 \%)$ \\
\hline Total & 17 & 22 & 21 & 7 & 3 & 6 & $\begin{array}{c}76(63.3 \\
\%)\end{array}$ \\
\hline
\end{tabular}

Most of the adverse outcomes were due to low grade temperatures. 


\begin{tabular}{|c|c|c|c|c|c|}
\hline Outcome & $\begin{array}{l}<=1 \\
\text { Week }\end{array}$ & $\begin{array}{c}8 \text { to } 14 \\
\text { Days }\end{array}$ & $\begin{array}{c}15 \text { to } 30 \\
\text { Days }\end{array}$ & > 30 Days & Total (\%) \\
\hline Abortion & 12 & 3 & 1 & 0 & $16(13.3 \%)$ \\
\hline IUGR & 0 & 2 & 1 & 4 & 7 (5.8 \%) \\
\hline PROM & 5 & 2 & 3 & 5 & 15 (12.5\%) \\
\hline Preterm labour & 1 & 4 & 2 & 5 & $12(10 \%)$ \\
\hline $\mathrm{PPH}$ & 2 & 0 & 0 & 1 & $3(2.5 \%)$ \\
\hline MSL. po & 4 & 0 & 0 & 2 & $6(5 \%)$ \\
\hline Neonatal death & 0 & 1 & 0 & 0 & $1(0.8 \%)$ \\
\hline Neonatal sepsis & 6 & 2 & 0 & 1 & $9(7.5 \%)$ \\
\hline IUD & 0 & 2 & 0 & 0 & $2(1.7 \%)$ \\
\hline Anomaly & 0 & 0 & 0 & 1 & $0(0 \%)$ \\
\hline Maternal sepsis & 3 & 0 & 0 & 0 & $3(2.5 \%)$ \\
\hline $\begin{array}{l}\text { Maternal } \\
\text { mortality }\end{array}$ & 1 & 0 & 0 & 0 & $1(0.8 \%)$ \\
\hline Total & 34 & 16 & 7 & 19 & $76(63.3 \%)$ \\
\hline \multicolumn{6}{|c|}{$\begin{array}{c}\text { Table 5. Adverse Outcomes and Interval from } \\
\text { the Onset of Fever to Adverse Outcome }\end{array}$} \\
\hline
\end{tabular}

With the interval between onset of fever and the outcome being less than 8 days, there were more adverse outcomes. This had significant $\mathrm{P}$-value $=0.03$.

\section{DISCUSSION}

There was a total of 120 patients included in this study. Of the 120 patients, $63(52.5 \%)$ patients recovered from fever and had no adverse maternal or fetal outcome. 57 (47.5\%) patients had adverse outcomes and of them 19 (15.8\%) had more than one adverse outcome. In this study, $26.7 \%$ of the patients had urinary tract infection as the aetiology of fever. The incidence is similar to the study by Joseph A.K (1972), in which the incidence was seen in $25 \%$ of pregnancies. Upadhaya et al. (1969) reported $20 \%$ of pregnant women and Collen (1979) reported $25.5 \%$. However, in other studies, Asha Deshpande (1985) - 13.5\%, Van Darsten (1986) - $10.2 \%$ and Jai Bhagwansharma (1990) - $12 \%$ have reported lower incidences of urinary tract infection in pregnancy. The most common organism involved was Escherichia coli, which was found in $67 \%$ of the patients with UTI. The predominance of E. coli between 48 to $85 \%$ has been reported by Roa et al. (1969), Carbtree (1972), Asha Deshpande (1985) and Michel. L. Lefevre (2000). In this study $10 \%$ of the cases had dengue fever and one patient had dengue haemorrhagic fever. As per Gibbons RV, Vaugh DW (2003), the estimated annual occurrence is 10 million cases and 250,000 cases of dengue haemorrhagic fever with a mortality rate of 25000 per year. 5 cases that occurred in the first trimester had an abortion. There were two cases of preterm labour and one case of premature rupture of membranes. Friedman EE et al. (2013) showed that $40 \%$ of the women with dengue fever had preterm deliveries with low birth weight babies, dengue fever at less than 17 weeks led to miscarriages. In this study there was one neonatal mortality due to transmission of dengue haemorrhagic fever from the mother to the fetus during delivery. In this study, malaria was found in $1.7 \%$ of the patients. One patient had an abortion and the other had an otherwise uneventful pregnancy. Park JE (1997) quoted an incidence of malaria in $3.4 \%$ of pregnant women. Konar H (2004) studied the manifestations of malaria in pregnancy and found that miscarriage (32.03\%), preterm labour (47.7\%), intrauterine growth restriction (IUGR) (1.8\%) had occurred. There was one case of chicken pox in the first trimester and it was found that the fetus had multiple fetal anomalies. Of the 5 cases of scrub typhus diagnosed, two patients had an abortion, and one patient had an intensive care unit (ICU) admission, the remaining two had uneventful outcomes. The most frequently seen adverse outcome in this study was abortion (13.3\%). However, Anne-Marie Nybo Anderson et al. (2002) showed $4.8 \%$ miscarriage and still births among women with fever in the first 16 weeks of pregnancy and concluded that there was no association between fever and fetal loss. In this study, the increased number of abortions could be due to the lower socioeconomic status and high incidence of referrals as it is a tertiary care centre. In this study there were 7 cases of intra uterine growth restriction (5.8\%). Intrapartum fever was associated with postpartum haemorrhage and meconium stained liquor. In this study there were 21 cases of intrapartum fever of which 3 women had postpartum haemorrhage (PPH) $(14.2 \%)$ and 7 women had meconium stained liquor (MSL) (33\%). Ola Malabarey et al. (2011) showed that fever due to chorioamnionitis (P-value 0.02) was significantly associated with PPH. Jeffrey M. Perlman, MB showed that maternal fever led to $25 \%$ cases of MSL and fetal heart rate abnormalities. In this study there was one maternal mortality and one ICU admission due to H1N1 influenza virus. In a study by Alicia M. Siston et al. (2010), which studied the pandemic of H1N1 influenza A virus among pregnant women, there was a maternal mortality of $5 \%$ and $22.6 \%$ of the women required ICU admission. There were 9 cases of neonatal sepsis with elevated c-reactive protein (CRP) levels in this study. They required a prolonged stay in neonatal intensive care units (NICU) and treatment with intravenous antibiotics. All 9 babies recovered well and were discharged without any complications. In this study, there were 12 cases of preterm labour $(10 \%)$ and 14 cases of premature rupture of membranes $(11.67 \%)$. In the study by Cunningham FG, Lucas MJ IN (1994), the association of preterm labour with untreated UTI was $30.6 \%$. Ajay Ghosh AC, Usha reported a similar incidence of $29 \%$ of preterm labour. However, the number of preterm deliveries was only $10-12 \%$ in both the studies.

\section{Summary}

Fever in pregnancy nearing term has been associated with adverse outcomes. Even elevated temperature in the first trimester can lead to fetal loss. Of the women with adverse outcomes, most of them had fever for less than five days duration. Hence, fever during pregnancy with duration of even less than one week in all trimesters can make an otherwise low risk pregnancy to a high risk. Most of the women had low grade temperatures and hence the same should not be neglected. In most of the women the fever was nonspecific. Of those women with a diagnosed aetiology, urinary tract infection was the most common. E. coli was the most common organism isolated. Other etiologies encountered were, dengue, malaria, scrub typhus, leptospirosis, enteric fever, H1N1 influenza A viral fever, respiratory tract infections and chicken pox. The most frequently seen adverse outcome in this study was abortion. There was one maternal mortality ( $0.8 \%)$ due to H1N 1 and one neonatal mortality ( $0.8 \%)$ due to dengue hemorrhagic fever which was transmitted to the fetus. There was one fetus with multiple anomalies and the mother had a history of chicken pox in the first trimester. There were 15 cases $(12.5$ $\%)$ of premature rupture of membranes and 12 (10\%) cases 
had preterm labour, however, none of them grew any organisms on high vaginal swab cultures. Intrapartum fever was associated with postpartum haemorrhage and meconium-stained liquor in some mothers. Large series over a longer period is needed to formulate institutional guidelines.

\section{CONCLUSIONS}

Fever during pregnancy can potentially lead to adverse maternal and perinatal outcomes even at a low-grade temperature of a short duration of less than a week. Even elevated temperature in the first trimester can lead to fetal loss. Fever in pregnancy nearing term has been associated with adverse perinatal outcomes. Some virulent organisms can lead to fetal anomalies and maternal death.

Data sharing statement provided by the authors is available with the full text of this article at jemds.com.

Financial or other competing interests: None.

Disclosure forms provided by the authors are available with the full text of this article at jemds.com.

\section{REFERENCES}

[1] Cracco C, Delafosse C, Baril L, et al. Multiple organ failure complicating probable scrub typhus. Clin Infect Dis 2000;31(1):191-2.
[2] Mahajan SK. Scrub typhus. J Assoc Physicians India 2005;53:954-8.

[3] Kim YS, Lee HJ, Chang $M$, et al. Scrub typhus during pregnancy and its treatment: a case series and review of the literature. Am J Trop Med Hyg 2006;75(5):955-9.

[4] Watt G, Walker DH. Scrub typhus. Chap. 52. In: Guerrant RL, Walker DH, Weller PF, eds. Tropical infectious diseases principles, pathogens and practice. Vol 1. $2^{\text {nd }}$ edn. Philadelphia, PA: Elsevier Churchill Livingstone 2006.

[5] Tseng BY, Yang HH, Liou JH, et al. Immunohistochemical study of scrub typhus: a report of two cases. Kaohsiung J Med Sci 2008;24(2):92-8.

[6] Suputtamongkol Y, Suttinont C, Niwatayakul K, et al. Epidemiology and clinical aspects of rickettsioses in Thailand. Ann N Y Acad Sci 2009;1166:172-9.

[7] Sharma PK, Ramakrishnan R, Hutin YJF, et al. Scrub typhus in Darjeeling, India: opportunities for simple, practical prevention measures. Trans R Soc Trop Med Hyg 2009;103(11):1153-8.

[8] Centers for Disease Control and Prevention (CDC). Maternal and infant outcomes among severely ill pregnant and postpartum women with 2009 pandemic influenza A (H1N1)--United States, April 2009-August 2010. MMWR Morb Mortal Wkly Rep 2011;60(35):11936. 